\title{
Die britische Ratspräsidentschaft 2005: Zwischen euro- päischen Erwartungen und innenpolitischen Restriktionen
}

\author{
Kai Oppermann*
}

Die Übernahme der sechsmonatigen Präsidentschaft des Rates der Europäischen Union macht mitgliedstaatliche Regierungen zu besonders exponierten Adressaten politischer Erwartungen sowohl auf europäischer als auch auf innenpolitischer Ebene. Auf beiden Ebenen wird die amtierende Präsidentschaft eng mit den Ergebnissen zwischenstaatlicher Verhandlungen im Rat assoziiert und für Fortschritte oder Rückschläge in diesen Verhandlungen verantwortlich gemacht. Der britische Ratsvorsitz sah sich einerseits hohen Erwartungen auf europäischer Ebene ausgesetzt, zur Überwindung der gegenwärtigen Krise des Integrationsprozesses beizutragen. Andererseits war der Handlungsspielraum der britischen Regierung, diesen Erwartungen gerecht zu werden, durch zahlreiche innenpolitische Restriktionen eingeschränkt. In diesem Spannungsfeld ist es der britischen Präsidentschaft je nach Politikfeld mit unterschiedlichem Erfolg gelungen, die Agenda der europäischen Integration durch eigene Impulse zu beeinflussen und in mitgliedstaatlichen Konflikten tragfähige Kompromisslösungen einzubringen.

\section{Die Ratspräsidentschaft im Zwei-Ebenen-Kontext}

In Folge ihrer herausgehobenen Positionierung im Entscheidungssystem der Europäischen Union steht die Präsidentschaft mehr noch als ,normale' Regierungen von Mitgliedstaaten unter dem Druck, die auf europäischer und innenpolitischer Ebene an sie gerichteten und potenziell widerstreitenden Anforderungen miteinander zu vereinbaren. Der strategische Handlungskontext einer Ratspräsidentschaft entspricht der Logik von Zwei-EbenenKonstellationen, nach der die Außenpolitik von Regierungen primär aus dem Imperativ folgt, die sich wechselseitig beeinflussenden internationalen und innerstaatlichen Anreize und Zwänge in Ausgleich zu bringen. ${ }^{1}$

Auf europäischer Ebene wird von einer Präsidentschaft in erster Linie erwartet, dass sie effektiv auf das Zustandekommen von Verhandlungslösungen im Rat hinarbeitet. ${ }^{2}$ Erstens gelten Präsidentschaften in dem Maße als erfolgreich, wie sie der Agenda im Rat durch eigene Initiativen und Prioritätensetzungen neue Impulse verleihen. Zweitens werden sie nach ihrer Fähigkeit beurteilt, durch die Vorlage von Kompromissvorschlägen und Paketlösungen aktiv zur Konsensfindung im zwischenstaatlichen Verhandlungsprozess beizutragen. ${ }^{3}$

Allerdings ist dieser Bewertungsmaßstab für eine Präsidentschaft auf europäischer Ebene nicht notwendig deckungsgleich mit den Erwartungen, die innenpolitisch an sie gerichtet werden. Innerstaatliche Akteure evaluieren die Performanz ihrer Regierung als Ratsvorsitz

* Dipl.-Pol. Kai Oppermann, Wissenschaftlicher Mitarbeiter am Lehrstuhl für Internationale Politik und Außenpolitik der Universität zu Köln. Ich danke Thomas Jäger, Anna Daun und Alexander Höse für wertvolle Anregungen und Kritik.

1 Vgl. Robert D. Putnam: Diplomacy and Domestic Politics: The Logic of Two-Level-Games, in: International Organization, 3/1988, S. 427-461.

2 Zu den Funktionen der Präsidentschaft vgl. ausführlich Fiona Hayes-Renshaw/Helen Wallace: The Council of Ministers, New York 1997, S. 139-150.

3 Vgl. Ole Elgström: Introduction, in: Ole Elgström (Hrsg.): European Union Council Presidencies: A Comparative Perspective, London 2003, S. 5-7. 
vor allem danach, inwieweit sie die von ihnen artikulierten Präferenzen wahrt und befördert. ${ }^{4}$ Das Erfordernis der innerstaatlichen Legitimation von Europapolitik ist für Regierungen während ihrer Amtszeit als Ratspräsidentschaft besonders stark ausgeprägt, weil damit eine erhöhte mediale und öffentliche Aufmerksamkeit für diese Politik einhergeht. Gerade der sukzessive Bedeutungszuwachs des Europäischen Rates und dessen Entwicklung zu einem Medienereignis ersten Ranges hat die innenpolitische Sichtbarkeit der Präsidentschaft zusätzlich erhöht. ${ }^{5}$

Aus der theoretischen Perspektive des Zwei-Ebenen-Ansatzes muss die traditionelle Sicht der Rolle der Präsidentschaft als neutraler und desinteressierter Makler somit verworfen werden. ${ }^{6}$ Diese Sichtweise beschreibt einen im wissenschaftlichen wie politischen Diskurs tief verankerten normativen Anspruch an den Ratsvorsitz, eignet sich jedoch nicht als analytischer Ausgangspunkt für die Bewertung individueller Präsidentschaften. ${ }^{7}$ Vielmehr stehen Regierungen in ihrer Funktion als Ratspräsidentschaft grundsätzlich vor der Notwendigkeit, die auf europäischer und auf innerstaatlicher Ebene an sie gerichteten Erwartungen gegeneinander abzuwägen. In Abhängigkeit von der politischen Konstellation auf beiden Ebenen ergeben sich unterschiedliche Ausgangsbedingungen für jede nationale Präsidentschaft.

Eine Analyse des britischen Ratsvorsitzes muss daher in einem ersten Schritt dessen europäische und innerstaatliche Handlungsbedingungen erfassen. Erst auf dieser Grundlage sind in einem zweiten Schritt Aussagen darüber möglich, mit welchem Erfolg es ihm gelungen ist, die Agenda der europäischen Integration durch eigene Impulse voranzutreiben und Kompromisslösungen für mitgliedstaatliche Konflikte zu erarbeiten. Dabei können Ratspräsidentschaften auf zwei Arten politischer Ressourcen zurückgreifen, um die auf beiden Ebenen formulierten Erwartungen zu verarbeiten. Dank der Zuarbeit des Generalsekretariates des Rates und in Folge bilateraler Konsultationen mit den übrigen mitgliedstaatlichen Regierungen profitieren sie erstens von einem Informationsvorsprung über die Verhandlungskonstellation auf europäischer Ebene. Zweitens besitzen Präsidentschaften vielfältige Möglichkeiten der Steuerung des politischen Prozesses auf europäischer Ebene, beispielsweise in dem sie die Frequenz, das Format und die Agenda der Sitzungen des Rates bestimmen oder in dem sie durch das Schnüren von Paketlösungen den Ausgangspunkt zwischenstaatlicher Verhandlungen definieren. ${ }^{8}$

4 Vgl. Jonas Tallberg: The agenda-shaping powers of the EU Council Presidency, in: Journal of European Public Policy, 1/2003, S. 4-5.

5 Zur Entwicklung und Bedeutung des Europäischen Rates vgl. Wolfgang Wessels: Das politische System der EU, in: Wolfgang Ismayr: Die politischen Systeme Westeuropas, 3. Aufl., Opladen 2003, S. 785-787; Peter Ludlow: Die Führung der Europäischen Union durch den Europäischen Rat: Übergang oder Krise?, in: integration, 1/05, S. 3-15.

6 Diese analytische Perspektive knüpft an jüngere Forschungsergebnisse an, wonach Präsidentschaften im Allgemeinen nicht als ,ehrliche“ Makler agieren, sondern eigene Präferenzen durchzusetzen versuchen. Vgl. Jonas Tallberg: The Power of the Presidency: Brokerage, Efficiency and Distribution in EU Negotiations, in: Journal of Common Market Studies, 5/2004, S. 1019-1020.

7 An prominentester Stelle ist dieser Anspruch im Handbuch des Generalsekretariates des Rates über die Präsidentschaft formuliert: ,The Presidency must, by definition, be neutral and impartial. It is the moderator for discussions and cannot therefore favour either its own preferences or those of a particular Member State." Vgl. General Secretariat: Council Guide, Vol. I, The Presidency Handbook, Luxemburg 2001, S. 5. Einen Überblick über die Verankerung dieser Sichtweise im wissenschaftlichen Diskurs bietet Ole Elgström: 'The Honest Broker'? The Council Presidency as a Mediator, in: Ole Elgström (Hrsg.): European Union Council Presidencies: A Comparative Perspective, London 2003, S. 39-41.

8 Vgl. Tallberg: Power of the Presidency, 2004, S. 1001-1005; David Metcalfe: Leadership in European Union Negotiations: The Presidency of the Council, in: International Negotiation, 3/1998, S. 422-426. 


\section{Die europäischen und innenpolitischen Handlungsbedingungen}

Der europäische Handlungskontext der britischen Ratspräsidentschaft war vor allem durch die aktuelle Krise des Integrationsprozesses geprägt. Nach den gescheiterten Referenden über die Europäische Verfassung in Frankreich und den Niederlanden markiert der Ratsvorsitz Großbritanniens den Auftakt der Reflexionsphase, die sich die europäischen Staatsund Regierungschefs im Hinblick auf die Zukunft der Verfassung verordnet haben. Das zumindest vorläufige Scheitern dieses Vertiefungsprojektes verschärfte die Auseinandersetzungen innerhalb der Union über die in den Bevölkerungen einiger Mitgliedstaaten mehrheitlich ablehnend beurteilte Fortsetzung des Erweiterungsprozesses insbesondere hinsichtlich eines Beitritts der Türkei. ${ }^{9}$ Zugespitzt hatte sich die krisenhafte Entwicklung der europäischen Integration schließlich dadurch, dass es auf dem Europäischen Rat vom 16./ 17. Juni 2005 nicht gelungen war, eine Einigung über die Finanzielle Vorausschau für die Jahre 2007 bis 2013 herbeizuführen. ${ }^{10}$ Die Verhandlungen endeten mit wechselseitigen Schuldzuweisungen, die in dieser Schärfe nur selten an die Öffentlichkeit gelangen und Ausdruck der starken zwischenstaatlichen Spannungen in dieser Frage waren.

Die britische Präsidentschaft stand vor diesem Hintergrund auf europäischer Ebene unter besonderem Erwartungsdruck, dem Integrationsprozess die gerade in Zeiten der Krise erforderliche politische Führung zuteil werden zu lassen. Im Konkreten wurden der britischen Regierung von ihren europäischen Partnern zwei explizite Prüfsteine angetragen: Ihre Präsidentschaft würde sich daran messen lassen müssen, ob es ihr gelingt, erstens den planmäßigen Beginn der Beitrittsverhandlungen mit der Türkei sicherzustellen und zweitens eine Vereinbarung über die Finanzielle Vorausschau der Europäischen Union herbeizuführen.

Diese hohen europäischen Erwartungen hat sich die britische Regierung von Beginn ihrer Präsidentschaft an zu Eigen gemacht. ${ }^{11}$ Darüber hinaus nutzte Tony Blair seine Rede vor dem Europäischen Parlament am 23. Juni 2005, um die Krise des Integrationsprozesses als Chance und Verpflichtung für eine umfassende Debatte über die zukünftige Gestaltung der Europäischen Union zu interpretieren, die unter Führung der britischen Präsidentschaft begonnen werden müsse. ${ }^{12}$ Es war nicht zuletzt diese sehr ambitionierte Rede, die den Erwartungsdruck auf die britische Regierung zusätzlich erhöhte und in der Folge häufig als Referenzpunkt für die Kritik an den tatsächlichen Fortschritten der Präsidentschaft zitiert wurde. Darin offenbart sich eine gewisse Parallelität zum ersten Ratsvorsitz New Labours in der ersten Jahreshälfte 1998, der dafür kritisiert wurde, überzogene Ansprüche formuliert und unrealistische Erwartungen geweckt zu haben. ${ }^{13}$

Der innenpolitische Kontext der britischen Präsidentschaft zeichnet sich in vielen Bereichen dadurch aus, dass er ihren Handlungsspielraum auf europäischer Ebene relativ eng begrenzt hat. Erstens haben die traditionell europaskeptische öffentliche Meinung und die mehrheitlich ebenfalls europaskeptisch mobilisierende Presse als langfristige Restriktionen britischer Europapolitik die Fähigkeit New Labours beschnitten, innenpolitische Zustimmung für europapolitische Initiativen und Kompromisspakete zu gewinnen. ${ }^{14}$

9 Vgl. Eurobarometer 64, Dezember 2005, Frage 32.4, erste Ergebnisse abrufbar unter: http://europa.eu.int/ comm/public_opinion/archives/eb/eb64/eb64_first_de.pdf (letzter Zugriff: 10.1.2006).

10 Vgl. Schlussfolgerungen des Vorsitzes, Europäischer Rat, 16./17.6.2005, 10255/1/05, Punkt 5.

11 Vgl. The UK Presidency of the European Union: Prospects for the EU in 2005, Cm 6611, June 2005, S. 6-8.

12 Vgl. Tony Blair: Speech to the European Parliament, 23.6.2005, abrufbar unter: http://www.pm.gov.uk/output/ Page7714.asp (letzter Zugriff: 29.12.2005).

13 Vgl. Peter Ludlow: The 1998 UK Presidency: A View from Brussels, in: Journal of Common Market Studies, 4/1998, S. 580-581; Douglas Henderson: The UK Presidency: An Insider's View, in: Journal of Common Market Studies, 4/1998, S. 564.

14 Vgl. Andrew Geddes: The European Union and British Politics, Houndmills, Basingstoke 2004, S. 211-224. 
Zweitens bestätigte sich im Juni 2005 das Potenzial der Europapolitik, zu einem der zentralen Bezugspunkte der politischen Debatte in Großbritannien zu werden. Im Zuge der Kontroverse über den britischen Beitragsrabatt stieg das öffentliche Interesse an dem Thema sprunghaft an. So wurde die Europapolitik in diesem Monat von 19 Prozent (Vormonat: 9 Prozent) der Befragten als eines der wichtigsten Probleme der politischen Agenda benannt. Sie gehörte damit zu den fünf Problemfeldern, die in der britischen Öffentlichkeit die größte Aufmerksamkeit genossen. ${ }^{15}$ Dieser Befund ist ein weiterer Beleg für die starke Sensibilität der britischen Öffentlichkeit gegenüber außergewöhnlichen Entwicklungen auf europäischer Ebene, die bereits in der Vergangenheit regelmäßig starke Aufschwünge des öffentlichen Interesses an europapolitischen Fragen angestoßen haben. ${ }^{16}$ Die britische Regierung konnte somit nicht davon ausgehen, ihre Rolle als Ratsvorsitzende hinter einem Schleier der öffentlichen Indifferenz ausfüllen zu können.

Vor diesem Hintergrund musste die britische Präsidentschaft ihre Politik drittens mit Blick auf ihre Rückwirkungen in die zwischenparteiliche Auseinandersetzung mit der konservativen Opposition formulieren. Die Europapolitik bietet der europaskeptisch positionierten konservativen Partei eine attraktive Möglichkeit, sich im Wettbewerb um wahlpolitische Zustimmung von der pro-europäischeren Programmatik New Labours abzugrenzen. ${ }^{17}$ Dementsprechend ist die Regierung Blair seit ihrem Amtsantritt bestrebt, der Opposition möglichst geringe europapolitische Angriffsflächen zu bieten. In diesem Sinne waren für die britische Präsidentschaft solche Schwerpunktsetzungen opportun, von denen sie nicht erwarten musste, dass sie die europaskeptische Kritik der konservativen Partei mobilisieren würden. Dazu gehören vor allem die Fortsetzung des Erweiterungsprozesses der Europäischen Union, die Reform der gemeinsamen Agrarpolitik oder die wirtschaftspolitische Liberalisierung und Deregulierung Europas. ${ }^{18}$

Viertens sieht sich die Regierung Blair nach ihrer Wiederwahl im Mai 2005 einer sukzessiven Schwächung ihres parlamentarischen Rückhaltes gegenüber. In Anbetracht der schon seit New Labours zweiter Amtszeit erodierenden Fraktionsdisziplin unter den Labour-Abgeordneten im Unterhaus erscheint die auf nur noch 66 Sitze reduzierte Regierungsmehrheit als zunehmend fragile Machtbasis. Dies fand seinen bislang dramatischsten Ausdruck in der Unterhausabstimmung am 9. November 2005 über einen Regierungsentwurf im Rahmen der Antiterrorgesetzgebung, als 49 Labour-Abgeordnete gegen die eigene Regierung stimmten und ihr damit die erste Abstimmungsniederlage ihrer achtjährigen Amtszeit beibrachten. ${ }^{19}$ Dieser innerparteiliche Widerstand gegen die Regierungspolitik bringt einen rapiden Autoritätsverlust Tony Blairs gegenüber der eigenen Fraktion zum Ausdruck. Mit seiner Ankündigung, sich noch vor den nächsten Wahlen vom Amt des Premierministers zurückzuziehen, hat Blair erheblich an politischen Ressourcen zur Disziplinierung der Labour-Abgeordneten verloren, deren Loyalitäten sich in Richtung seines potenziellen Nachfolgers Gordon Brown verschoben haben.

Im Ergebnis befanden sich der europäische und der innenpolitische Handlungskontext der britischen Präsidentschaft in einem Spannungsverhältnis zueinander. Die britische Re-

15 Vgl. MORI Political Monitor, Recent Trends: The Most Important Issues Facing Britain Today, abrufbar unter: http://www.mori.com/polls/trends/issues12.shtml (letzter Zugriff: 29.12.2005).

16 Vgl. Kai Oppermann: Der europapolitische Wandel der Labour-Partei, in: André Kaiser/Sebastian Berg (Hrsg.): New Labour und die Modernisierung Großbritanniens, Augsburg 2006 (im Erscheinen).

17 Vgl. Philip Cowley/Stuart Quayle: The Conservatives: Running on the spot, in: Andrew Geddes/Jonathan Tonge (Hrsg.): Labour's Second Landslide: The British General Election 2001, Manchester 2002, S. 57-58.

18 Vgl. Michael Howard: Statement to the House of Commons, in: House of Commons Hansard Debates, 20.6.2005, vol. 435 , cols. 525-527.

19 Vgl. Philip Webster: „Beginning of the end?“, in: The Times, 10.11.2005, S. 1. 
gierung stand vor dem schwierigen Spagat, die auf europäischer Ebene an sie gerichteten hohen Erwartungen mit den engen Restriktionen ihres Handlungsspielraums auf innerstaatlicher Ebene in Einklang zu halten. Die nachfolgenden Abschnitte sollen zeigen, inwieweit es dem britischen Ratsvorsitz in dieser Konstellation gelungen ist, erstens die Agenda der europäischen Integration durch eigene Impulse zu beeinflussen und zweitens zur Vermittlung mitgliedstaatlicher Konflikte beizutragen.

\section{Der britische Einfluss auf die europäische Agenda}

Nationale Regierungen nutzen ihre politischen Ressourcen als Ratspräsidentschaft, um die Agenda der europäischen Integration nach eigenen Prioritäten zu prägen. Diese Einflussnahme bezieht sich nicht ausschließlich darauf, auf europäischer Ebene genuin neue Themen zu etablieren. Gerade weil Regierungen bei Übernahme der Präsidentschaft in der Regel eine dicht gestaffelte Agenda nicht abschließend abgearbeiteter Themen vorfinden, manifestiert sich ihr Einfluss vor allem darin, einige dieser Themen mit besonderem Nachdruck in den Vordergrund zu rücken oder sie umgekehrt nicht aktiv zu behandeln und damit zumindest vorläufig von der Tagesordnung zu nehmen. ${ }^{20}$

Zunächst bietet die Politik des britischen Ratsvorsitzes hinsichtlich der Europäischen Verfassung ein idealtypisches Beispiel für die Möglichkeiten einer Präsidentschaft, bestimmte Themen aus der offiziellen Diskussion im Rat auszuklammern. So hat die Regierung Blair die im Juni 2005 vereinbarte Phase der Reflexion über die Verfassung als Rechtfertigung genutzt, um diese Reflexion in das Jahr 2006 zu verschieben und nicht unter ihrem Vorsitz auf die Tagesordnung zu setzen. ${ }^{21}$ Damit vermied sie eine erneute innenpolitische Debatte über die Verfassung, die von einer breiten Mehrheit der britischen Öffentlichkeit, von der konservativen Opposition und von signifikanten Teilen der Labour-Partei abgelehnt wird. Die von verschiedener Seite geäußerte Kritik an dieser Politik und die Ankündigung der neuen deutschen Bundesregierung, ihre Präsidentschaft in der ersten Jahreshälfte 2007 dafür zu nutzen, die Europäische Verfassung zurück in das Zentrum der europäischen Agenda zu rücken, stützt das kontrafaktische Argument, dass die britische Haltung nicht alternativlos war, sondern dass eine Präsidentschaft mit einer anderen Interessenlage dazu hätte beitragen können, die Debatte über die Zukunft der Verfassung schon im Jahre 2005 voranzutreiben. ${ }^{22}$ Der britische Ratsvorsitz besaß in der Frage der Europäischen Verfassung einen diskretionären Handlungsspielraum, den er mit einer Politik der Nichtentscheidung, also der intentionalen Beschränkung des thematischen Raumes politischer Entscheidungen, zur Beeinflussung der europäischen Agenda nutzte. ${ }^{23}$

Im Gegensatz dazu sind drei Themenbereiche auszumachen, welche die Regierung Blair mit besonderem Nachdruck verfolgt hat. ${ }^{24}$ Erstens erhob sie die Diskussion über eine Reform der europäischen Sozial- und Wirtschaftspolitik unter den Bedingungen der Globalisierung von Beginn an zur obersten Priorität ihrer Präsidentschaft. ${ }^{25}$ Dieser Fokus steht in der

20 Zur konzeptionellen Unterscheidung zwischen agenda-setting, agenda-structuring und agenda-exclusion vgl. Tallberg: Agenda-shaping powers, 2003, S. 4-13.

21 Vgl. The UK Presidency of the European Union: Prospects, 2005, S. 6.

22 Vgl. Quentin Peel: ,A presidency in danger of irrelevance“, in: Financial Times, 24.11.2005, S. 21; Koalitionsvertrag zwischen CDU, CSU und SPD: Gemeinsam für Deutschland - mit Mut und Menschlichkeit, 11.11.2005, S. 127.

23 Vgl. Peter Bachrach/Morton S. Baratz: Decisions and Non-decisions: An Analytical Framework, in: American Political Science Review, 3/1963, S. 632-634.

24 Vgl. UK Presidency of the EU 2005: Priorities for the UK Presidency of the EU 2005, abrufbar unter: http:// www.eu2005.gov.uk/servlet/Front?pagename $=$ OpenMarket/Xcelerate/ ShowPage\&c=Page\&cid=1107293521089 (letzter Zugriff: 29.12.2005).

25 Vgl. Tony Blair: Speech to the European Parliament, 23.6.2005. 
Kontinuität der bisherigen Europapolitik New Labours, die solche Reformen bereits zum Ziel ihres ersten Ratsvorsitzes im Jahre 1998 erklärte und sich in der Zwischenzeit als treibende Kraft hinter der Reformagenda des Lissabon-Prozesses profilierte. ${ }^{26}$

Ihren augenfälligsten Ausdruck fand diese Schwerpunktsetzung in dem Treffen der Staats- und Regierungschefs von Hampton Court am 27. Oktober 2005. Als einzigen Tagesordnungspunkt des informellen Europäischen Rates benannte die britische Präsidentschaft die Chancen und Herausforderungen der Globalisierung. ${ }^{27}$ Dabei setzte sich die Regierung Blair über die breite Kritik ihrer europäischen Partner hinweg, die insbesondere eine Diskussion über die Finanzielle Vorausschau 2007-2013 einforderten. Der Gipfel von Hampton Court bestätigt damit die Fähigkeit nationaler Präsidentschaften, der europäischen Agenda kraft ihrer organisatorischen Ressourcen eigene Akzente zu verleihen.

Die konkreten Ergebnisse des Gipfels waren jedoch sehr begrenzt. Sie beschränkten sich im Wesentlichen darauf, dass die Präsidentschaft die breite Zustimmung der Staats- und Regierungschefs zu einem Konzeptpapier der Europäischen Kommission feststellen konnte, das den sozial- und wirtschaftspolitischen Reformbedarf Europas aufzeigt und verschiedene Anstöße für solche Reformen gibt. ${ }^{28}$ Dies galt jedoch nicht für den Vorschlag eines Globalisierungsfonds, der im Falle von Massenentlassungen als Folge des Wettbewerbsdrucks in einer globalisierten Wirtschaft Gelder zur Reintegration der Betroffenen in den Arbeitsmarkt bereitstellen sollte. Der britische Vorsitz unterstützte die Initiative zwar als taktisches Instrument, um der französischen Regierung Zugeständnisse bei der Reform der gemeinsamen Agrarpolitik zu erleichtern, jedoch wurde der Fonds von den großen Nettozahlern in den EU-Haushalt abgelehnt. ${ }^{29}$

Neben diesen öffentlichkeitswirksamen Beratungen auf höchster politischer Ebene hat die Präsidentschaft ihre wirtschafts- und sozialpolitische Agenda dadurch verfolgt, dass sie auf die Verabschiedung verschiedener Richtlinien hingearbeitet hat. Die bedeutendste und kontroverseste dieser Richtlinien ist ohne Zweifel die Dienstleistungsrichtlinie. Die britische Regierung hat es als Ziel ihres Vorsitzes definiert, den unter luxemburgischer Präsidentschaft ohne Ergebnis gebliebenen Verhandlungen neue Impulse zu verleihen und die Konsensfindung im Rat voranzutreiben. ${ }^{30}$ Zwar wurde der Prozess der Gesetzgebung nach dem Mitentscheidungsverfahren weitergeführt, so dass das Europäische Parlament voraussichtlich im Februar 2006 in erster Lesung über einen modifizierten Richtlinienentwurf beschlieBen wird. ${ }^{31}$ Das britische Bemühen um eine Annäherung der Standpunkte im Rat ist letztlich jedoch ohne entscheidenden Erfolg geblieben, da es die Ablehnung zentraler Aspekte der Richtlinie insbesondere durch die französische Regierung nicht zu überwinden vermochte. Ebenso wenig Erfolg war der britischen Absicht beschieden, die Verhandlungen über eine Neufassung der Arbeitszeitrichtlinie unter ihrer Präsidentschaft zum Abschluss zu bringen. ${ }^{32}$

26 Vgl. Wyn Grant: Economic Policy, in: Patrick Dunleavy/Andrew Gamble/Richard Heffernan/Gillian Peele (Hrsg.): Developments in British Politics 7, Houndmills, Basingstoke 2003, S. 264-267.

27 Vgl. UK Presidency of the EU 2005: Letter of Invitation to Informal meeting of Heads of State and Government at Hampton Court on 27 October from the Prime Minister, abrufbar unter: http://www.eu2005.gov.uk/ servlet/Front?pagename=OpenMarket/Xcelerate/ShowPage $\& c=$ Page \&cid=1107293561746\&a $=$ KAricle \&aid $=$ 1129040976862 (letzter Zugriff: 29.12.2005).

28 Vgl. European Commission: European values in the globalised world, Contribution of the Commission to the October Meeting of Heads of State and Government, Com (2005) 525, 20.10.2005, Brussels.

29 Vgl. Anthony Browne: „Blair goes global to appease Paris over EU budget“, in: The Times, 26.10.2005, S. 32.

30 Vgl. The Federal Trust: The UK Presidency of the European Union 2005, European Policy Brief, 13, June 2005.

31 Vgl. Draft European Parliament Legislative Resolution, A6-0409/2005, abrufbar unter: http://www.entemp.ie/ trade/marketaccess/singlemarket/05serv597.pdf (letzter Zugriff: 10.1.2006).

32 Vgl. Jack Straw: Statement to the House of Commons, in: House of Commons Hansard Debates, 30.6.2005, vol. 435 , col. 1451. 
Dabei erwies sich gerade das Anliegen New Labours, auch weiterhin Ausnahmen von der wöchentlichen Höchstarbeitszeit von 48 Stunden zuzulassen, als größtes Hindernis für eine Einigung. ${ }^{33}$

Im Gegensatz dazu kann die britische Präsidentschaft bei zwei weiteren Schwerpunkten ihrer Reformagenda auf Ergebnisse verweisen. So gelangen erstens maßgebliche Fortschritte bei der Implementation und Weiterentwicklung des Aktionsplans Finanzdienstleistungen (FSAP). ${ }^{34}$ Zweitens hat der Rat die Kommission aufgefordert, ihre Initiativen für neue europäische Richtlinien und Verordnungen ab Januar 2006 auf die durch sie verursachten administrativen Kosten zu überprüfen und entspricht damit dem britischen Eintreten für einen Abbau regulativer Belastungen für Unternehmen auf europäischer Ebene. ${ }^{35}$ Da diese Fortschritte allerdings Bereiche betreffen, die zwischen den Mitgliedstaaten im Grundsatz wenig strittig waren, erscheint der tatsächliche Beitrag des britischen Vorsitzes dazu eher gering.

Neben der Wirtschafts- und Sozialpolitik kristallisierte sich die Antiterrorpolitik als zweite Priorität der Präsidentschaft heraus. Es waren nicht zuletzt die Terroranschläge vom 7. Juli 2005 in London, die diesem Politikfeld aus Sicht der Regierung Blair zusätzliche Dringlichkeit verliehen haben. In Folge der Anschläge wurde der internationale Terrorismus in der britischen Öffentlichkeit als das mit Abstand wichtigste politische Problem wahrgenommen. ${ }^{36}$ In dieser Konstellation bot der Ratsvorsitz New Labour eine zusätzliche Ressource, ihre Antiterrorpolitik auf innerstaatlicher Ebene durch eine europäische Dimension zu ergänzen und damit effektiver zu gestalten. Dass die britische Präsidentschaft in diesem Politikfeld mit besonderem Nachdruck versuchen würde, der europäischen Agenda neue Impulse zu verleihen, zeigte sich bereits am 13. Juli 2005, als sie eine Sondersitzung des Rates der Justiz- und Innenminister einberief, um zusätzliche Maßnahmen der Terrorbekämpfung zu diskutieren.

Im Ergebnis sind der britischen Präsidentschaft in diesem Feld zwei signifikante Erfolge zu bescheinigen. Erstens ist es ihr gelungen, die Zustimmung der übrigen Mitgliedstaaten zu ihrer Initiative für eine europäische Antiterrorstrategie zu gewinnen. Zweitens konnte der britische Vorsitz eine Vereinbarung auf europäische Mindestvorschriften zur Speicherung von Telekommunikationsdaten erreichen. Die Regierung Blair hat einer solchen Vereinbarung höchste Bedeutung für eine effektive Terrorbekämpfung beigemessen und dazu einen Vorschlag präsentiert, der jedoch zunächst auf Kritik einiger Mitgliedstaaten stieß. Durch verschiedene Abstriche von ihrem ursprünglichen Entwurf war die Präsidentschaft in der Lage, diese Kritik zu zerstreuen und Anfang Dezember einen Konsens herbeizuführen. ${ }^{37}$ Schließlich wurden auch in den Verhandlungen über die gegenseitige Anerkennung von Beweismitteln in Strafsachen Fortschritte erzielt, die von der Regierung Blair ebenfalls als zentrales Anliegen ihres Vorsitzes identifiziert wurden. ${ }^{38}$

33 Vgl. Provisional Conclusions: Employment, Social Policy, Health and Consumer Affairs Council, 8-9 December 2005, 15201/05, S. 11, abrufbar unter: http://www.fco.gov.uk/Files/kfile/eu2005_CC_ESPHCA_0809dec.pdf (letzter Zugriff: 29.12.2005).

34 Vgl. Economic and Financial Affairs Council Conclusions, 11 October 2005, 12800/05, S. 11-12, abrufbar unter: http://www.fco.gov.uk/Files/kfile/ECOFIN_11October_Conclusions.pdf (letzter Zugriff: 29.12.2005).

35 Vgl. Provisional Conclusions: Economic and Financial Affairs Council, 8 November 2005, 13678/05, S. 11, abrufbar unter: http://www.fco.gov.uk/Files/kfile/EcofinConclusions_08nov.pdf (letzter Zugriff: 29.12.2005).

36 Vgl. MORI Political Monitor, Recent Trends: The Most Important Issues Facing Britain Today.

37 Vgl. Justice and Home Affairs Council Conclusions, 1-2 December 2005, 14390/05, abrufbar unter: http:// www.fco.gov.uk/Files/kfile/JHA_Conclusions_1-2Dec.pdf (letzter Zugriff: 29.12.2005).

38 Vgl. Andy Burnham: Statement to the House of Commons European Standing Committee, in: House of Commons European Standing Committee Debates, 1.12.2005, cols. 3-5, abrufbar unter: http://www.publications.parliament.uk/pa/cm200506/cmstand/euro/st051201/51201s01.htm (letzter Zugriff: 29.12.2005). 
Ein dritter Schwerpunktbereich der britischen Präsidentschaft betraf die Außenbeziehungen der Union. ${ }^{39}$ Dabei versuchte die Regierung Blair der europapolitischen Agenda vor allem in dreierlei Hinsicht eine eigene Handschrift zu verleihen. Erstens nutzte sie den Ratsvorsitz, um das traditionelle britische Anliegen eines freihändlerischen Europas voranzutreiben. ${ }^{40}$ Insbesondere war die britische Regierung bestrebt, den diplomatischen Druck für einen erfolgreichen Abschluss der Welthandelsgespräche in Hong Kong zu erhöhen und im innereuropäischen Entscheidungsprozess vor allem Frankreich zu Zugeständnissen bei der Liberalisierung des europäischen Agrarmarktes zu bewegen. ${ }^{41}$ Damit gelang es dem britischen Ratsvorsitz, einen Konnex zwischen den Welthandelsgesprächen und den Auseinandersetzungen über die Finanzielle Vorausschau der Europäischen Union herzustellen und Verhandlungsmacht für ihr Anliegen einer Reform der gemeinsamen Agrarpolitik aufzubauen. Einen zählbaren Erfolg ihrer Freihandelsagenda, der zugleich einen wesentlichen Baustein zu der in Hong Kong erreichten Vereinbarung beisteuerte, konnte der britische Vorsitz zudem bei der Reform der Zuckermarktordnung verzeichnen. Nach kontroversen Verhandlungen verständigten sich die europäischen Agrarminister auf einen britischen Kompromissvorschlag, der die Verringerung des Garantiepreises für Zucker um insgesamt 36 Prozent über vier Jahre vorsieht. ${ }^{42}$

Zweitens forcierte die britische Präsidentschaft die entwicklungspolitische Agenda der Europäischen Union. Damit setzte die Regierung Blair auch auf europäischer Ebene einen inhaltlichen Schwerpunkt, der bereits ihren G8-Vorsitz ausgezeichnet hat und mit dem sie sich spätestens seit den letzten Unterhauswahlen innenpolitisch zu profilieren versucht. Der bedeutendste Erfolg in diesem Bereich war die Annahme eines von ihr initiierten Strategiepapiers zu den Beziehungen zwischen der Europäischen Union und Afrika, das unter anderem auf die Intensivierung des politischen Dialoges mit den afrikanischen Staaten abzielt. ${ }^{43}$

Schließlich setzte sich die Regierung Blair drittens aktiv für eine Stärkung der Rolle Europas bei der Friedenssicherung in verschiedenen Krisenregionen der Welt ein. Auch darin steht die Präsidentschaft New Labours in der Kontinuität britischer Europapolitik, nach der sich die Gemeinsame Außen- und Sicherheitspolitik (GASP) weniger an dem Aufbau neuer Institutionen, sondern in erster Linie an ihren militärischen Ressourcen und praktischen Erfolgen in konkreten Aktionen messen lassen soll. Obwohl der exakte Beitrag der britischen Regierung dazu nicht aufzuschlüsseln ist, kann sie als Ergebnis ihrer Amtszeit herausstellen, dass unter britischem Vorsitz insgesamt sieben Missionen der GASP in Gang gesetzt wurden. ${ }^{44}$

\section{Die britische Präsidentschaft als Konfliktlöser}

Neben ihrer Rolle als Impulsgeber der europäischen Integration stand die britische Präsidentschaft vor der Herausforderung, in mitgliedstaatlichen Konfliktkonstellationen aktiv zur

39 Vgl. UK Presidency of the EU 2005: Priorities for the UK Presidency of the EU 2005.

40 Vgl. Gordon Brown: Global Britain, Global Europe: A Presidency founded on Pro European Realism, Speech at the Mansion House, London, 22.6.2005, abrufbar unter: http://www.hm-treasury.gov.uk/newsroom_ and_speeches/press/2005/press_57_05.cfm (letzter Zugriff: 29.12.2005).

41 Vgl. Larry Elliott: „Blair to pledge ,monumental struggle' on trade“, in: The Guardian, 14.11.2005, S. 27.

42 Vgl. Provisional Conclusions: Agriculture and Fisheries Council, 22-24 November 2005, 14178/05, abrufbar unter: http://www.fco.gov.uk/Files/kfile/eu2005_CC_AgFish_22-24nov,0.pdf (letzter Zugriff: 29.12.2005).

43 Vgl. European Council Conclusions, 15-16 December 2005, 15914/05, abrufbar unter: http:// www.eu2005.gov.uk/servlet/Front?pagename=OpenMarket/Xcelerate/ShowPage\&c=Page\&cid=11072935617 46\&a=KArticle\&aid=1134648491679 (letzter Zugriff: 29.12.2005).

44 Vgl. General Affairs and External Relations Council Conclusions, 21-22 November 2005, 14172/05, abrufbar unter: http://www.fco.gov.uk/Files/kfile/GAERC_Ext_21Nov.pdf (letzter Zugriff: 29.12.2005). 
Konsensfindung beizutragen. Mit dem Disput über den Beginn der Beitrittsverhandlungen mit der Türkei sowie den Auseinandersetzungen über die Finanzielle Vorausschau 20072013 standen zwei besonders spannungsgeladene Fragen zur Entscheidung an, welche die Außenwahrnehmung der Präsidentschaft mehr geprägt haben als alle übrigen Themen.

\section{Die Beitrittsverhandlungen mit der Türkei}

Der erste große Konflikt zwischen den Mitgliedstaaten der Europäischen Union unter britischem Vorsitz betraf die Beitrittsverhandlungen mit der Türkei. Es fiel in die Verantwortung der britischen Präsidentschaft, einen Konsens über den Rahmen dieser Verhandlungen herbeizuführen, so dass der im Dezember 2004 vereinbarte Termin für ihren Beginn am 3. Oktober 2005 eingehalten werden konnte. ${ }^{45}$ Die britische Regierung gehört innerhalb der Europäischen Union zu den nachdrücklichsten Befürwortern der Beitrittsverhandlungen und betont dabei die strategische Bedeutung der Türkei für das weltpolitische Gewicht der Union und als Brücke in die Krisenregion des Mittleren Ostens. Zudem fungiere die Beitrittsperspektive als Katalysator für demokratische und rechtstaatliche Reformen in der Türkei und wirke als wichtiges Symbol für die Chancen der friedlichen Kooperation zwischen der christlichen und der islamischen Welt. ${ }^{46}$ Gestützt wird die Haltung der britischen Regierung durch ihren wichtigsten Verbündeten auf internationaler Ebene, den USA, die von der Europäischen Union seit Langem die Aufnahme der Türkei einfordern. ${ }^{47}$

Diese Politik New Labours steht in Einklang mit den innenpolitischen Anforderungen an ihre Europapolitik. Zwar wird ein Türkeibeitritt zur Europäischen Union auch in der britischen Öffentlichkeit von einer relativen Mehrheit kritisch beurteilt, ohne dass die Problematik jedoch eine starke Mobilisierungskraft für die öffentliche Meinung besitzt. ${ }^{48} \mathrm{Da}$ sich die Regierung Blair in ihrer Türkeipolitik der geschlossenen Unterstützung der eigenen Partei, der konservativen Opposition und selbst des europaskeptischen Segments der britischen Presse sicher sein konnte, wurde ihr Eintreten für die Aufnahme von Beitrittsverhandlungen nicht durch signifikante innenpolitische Restriktionen beeinträchtigt.

Allerdings erwies sich der Auftakt der Beitrittsverhandlungen auf europäischer Ebene als konfliktreicher als im Vorfeld erwartet worden war. Der Konflikt manifestierte sich in zwei Dimensionen, bei denen der britische Vorsitz im Ergebnis entscheidend zu Kompromisslösungen beitragen konnte. Die erste Konfliktdimension stellte sich als Interessengegensatz zwischen der Türkei und der Republik Zypern dar. Ausgangspunkt war dabei die explizite Weigerung der türkischen Regierung, das EU-Mitglied Zypern diplomatisch anzuerkennen und die Zollunion zwischen der Türkei und der Europäischen Union auch im Verhältnis zu Zypern zu implementieren. Im Gegenzug forderte die zypriotische Regierung eine Erklärung der Europäischen Union, die der Türkei einen Zeitplan für die Normalisierung der Beziehungen zwischen den beiden Staaten vorgeben sollte. Die türkische Regierung lehnte eine derartige Erklärung als inakzeptabel ab und drohte ihrerseits mit Abbruch des Beitrittsprozesses. In dieser Konstellation erreichte die Präsidentschaft einen Konsens über eine abge-

45 Zur europäischen Erweiterungspolitik hinsichtlich der Türkei vgl. Barbara Lippert: Die Türkei als Sonderfall und Wendepunkt der klassischen EU-Erweiterungspolitik, in: integration, 1/05, S. 119-135.

46 Vgl. Jack Straw: Bridging the Bosporus - Turkey's European Future, Speech at the Institute of Public Policy Ressearch, London, 8.9.2005, abrufbar unter: http://www.eu2005.gov.uk/servlet/Front?pagename=OpenMarket/Xcelerate/ShowPage \&c=Page \&cid=1115146994906\&a=KArticle\&aid=1125560522451\&date=2005-0908 (letzter Zugriff: 29.12.2005).

47 Vgl. Stephen Castle: „Britain warns of Islamic Backlash if EU snubs Turkey“, in: The Independent, 9.9.2005, S. 27.

48 Vgl. YouGov: Survey on Britain, Europe and the G8, 20.-21.6.2005, abrufbar unter: http://www.yougov.com/ archives/pdf/RMW050101026_1.pdf (letzter Zugriff: 29.12.2005). 
milderte Erklärung der Union, in der die Anerkennung sämtlicher Mitgliedstaaten durch die Türkei als notwendige Komponente des Beitrittsprozesses benannt wird, ohne einen expliziten Zeitrahmen zu formulieren, und die von der türkischen Regierung eine vollständige Implementation ihrer Verpflichtungen hinsichtlich der Zollunion bis 2006 verlangt. ${ }^{49}$ Dabei war es eine wertvolle Ressource des britischen Ratsvorsitzes, dass er in Ankara dank seiner festen Unterstützung der türkischen Beitrittsperspektive als glaubwürdiger Gesprächspartner akzeptiert wurde und türkische Bedenken gegen die Erklärung der Europäischen Union daher ausräumen konnte.

Die zweite Dimension des Konfliktes über die Beitrittsperspektive der Türkei beruhte auf der Forderung Österreichs, einen Beitritt der Türkei zur Union im Verhandlungsmandat der Kommission nicht als ausschließliches Ziel der Verhandlungen festzuschreiben, sondern explizit auf mögliche Alternativen unterhalb der Schwelle einer Vollmitgliedschaft zu verweisen. ${ }^{50}$ Mit dieser Forderung ging die österreicherische Regierung hinter bestehende Beschlüsse der Union zurück und sah sich gegenüber den übrigen Mitgliedstaaten weitgehend isoliert. Die türkische Regierung weigerte sich, in einen Verhandlungsprozess einzutreten, der nicht ausdrücklich auf das Ziel einer Vollmitgliedschaft ausgerichtet ist.

Die britische Präsidentschaft konnte diese Blockade überwinden, indem sie erstens den Verhandlungsdruck auf die österreichische Regierung in bilateralen Gesprächen und durch die Einberufung einer Dringlichkeitssitzung des Rates auf Außenministerebene massiv erhöhte. Zweitens bot sie Österreich an, in den Verhandlungsrahmen einen expliziten Bezug auf die Aufnahmefähigkeit der Europäischen Union als Voraussetzung für einen Beitritt der Türkei aufzunehmen. Dieses Zugeständnis war in der Substanz zwar ohne Bedeutung, weil es lediglich eines der etablierten Kopenhagener Kriterien von 1993 bestätigt. Politisch sollte es der österreicherischen Regierung eine Brücke bauen, um den Beitrittsverhandlungen zustimmen zu können. Drittens nutzte der britische Vorsitz den Bericht der Chefanklägerin beim internationalen Kriegsverbrechertribunal in Den Haag, Carla del Ponte, vom 3. Oktober 2005, welcher der kroatischen Regierung ihre volle Kooperation mit dem Tribunal bescheinigte, um einen engen zeitlichen Zusammenhang zwischen dem Beginn von Beitrittsverhandlungen mit der Türkei und mit Kroatien herzustellen. Dieser Bericht machte den zentralen Hinderungsgrund für die Aufnahme von Beitrittsverhandlungen mit Kroatien gegenstandslos, die von Österreich stark befürwortet wurden. Die Parallelität zwischen den Verhandlungen über diese Themen hat der österreichischen Regierung einen starken Anreiz gegeben, von ihren Forderungen hinsichtlich der türkischen Beitrittsperspektive abzurücken. ${ }^{51}$

\section{Die Finanzielle Vorausschau 2007-2013}

Den größten mitgliedstaatlichen Konflikt während ihrer Präsidentschaft hatte die britische Regierung jedoch in den Verhandlungen über die Finanzielle Vorausschau der Europäischen Union für die Jahre 2007 bis 2013 zu bewältigen. Bei keinem anderen Thema standen die Erwartungen an den britischen Vorsitz auf europäischer Ebene in einem ähnlich starken

49 Vgl. Declaration by the European Community and its Member States in response to the declaration by Turkey made at the time of signature of the Additional Protocol to the Ankara Agreement, 21.9.2005, abrufbar unter: http://www.eu2005.gov.uk/servlet/Front?pagename=OpenMarket/Xcelerate/ShowPage\&c=Page\&cid=111514 6994806\&a=KArticle\&aid=1125564104376\&date=2005-09-21 (letzter Zugriff: 29.12.2005).

50 Vgl. Charles E. Ritterband: „Die Türken vor und in Wien: Österreichs eigenwillige EU-Position in der Türkeifrage“, in: Neue Zürcher Zeitung, 27.9.2005, S. 9.

51 Vgl. Nicholas Watt/Helena Smith: „Ankara and the EU: Sweet and sour climax to Turkey's long march“, in: The Guardian, 4.10.2005, S. 14. 
Spannungsverhältnis zu dessen innenpolitischen Restriktionen. Nach dem Auftakt der Beitrittsverhandlungen mit der Türkei wurde eine Vereinbarung über die Finanzielle Vorausschau noch im Jahre 2005 auf europäischer Ebene als entscheidender Beitrag zur Überwindung der europäischen Integrationskrise betrachtet. Entsprechend scharf war die Kritik, die in zahlreichen europäischen Hauptstädten und von der Kommission an der Verhandlungsführung der britischen Regierung geäußert wurde und die ihr mangelnde Kompromissbereitschaft, unzureichendes Engagement bei der Konsenssuche und die zu späte Vorlage konkreter Textgrundlagen für die Verhandlungen zum Vorwurf machte. ${ }^{52}$ Auf innerstaatlicher Ebene stand New Labour unter großem politischem und öffentlichem Druck, in den Finanzverhandlungen keine wesentlichen Zugeständnisse zu machen, die zu einer Mehrbelastung Großbritanniens führen würden.

Die Konfliktkonstellation auf europäischer Ebene wurde von vier Themenkomplexen dominiert, die zueinander in einem Nullsummenverhältnis standen und daher eine Verhandlungslösung stark erschwerten. Erstens war das Gesamtvolumen der Finanziellen Vorausschau strittig. In seinem Kompromisspapier vom 5. Dezember schlug der britische Vorsitz eine Verringerung der Gesamtausgaben der Union um etwa 24 Milliarden Euro im Vergleich zu dem im Juni gescheiterten Entwurf der luxemburgischen Präsidentschaft vor und bediente damit die Interessen der Nettozahler in den europäischen Haushalt. ${ }^{53}$ Den Großteil des anvisierten Sparvolumens wollte die britische Präsidentschaft zweitens bei den Strukturfondsmitteln für die neuen Mitgliedstaaten in Mittel- und Osteuropa realisieren. So wies ihr erster Entwurf für die Finanzielle Vorausschau gegenüber dem luxemburgischen Vorschlag eine Kürzung dieser Mittel um insgesamt 14 Milliarden Euro aus. Von den mittel- und osteuropäischen Staaten erwartete die britische Regierung eine weit gehende Kompromissbereitschaft, weil diese ein fundamentales Interesse an einer europäischen Verhandlungslösung besaßen, ohne die sich ihr Zugriff auf die vorgesehenen Fördergelder über den geplanten Zeitrahmen hinaus verzögert hätte, und die sich folglich in einer schwachen Verhandlungsposition befanden. Allerdings waren die beabsichtigen Kürzungen so umfangreich, dass vor allem die polnische Regierung, die allein auf sechs Milliarden Euro der versprochenen Subventionen verzichten sollte, den britischen Vorschlag schon aus innenpolitischen Gründen ablehnen musste.

Drittens trat der britische Vorsitz für strukturelle Veränderungen des europäischen Haushaltes ein, die zu einer langfristigen Absenkung der Agrarausgaben führen sollten. Entgegen einer Vereinbarung vom Oktober 2002 verlangte die britische Regierung eine umfassende Überprüfung der Agraraufwendungen bereits im Jahre 2008. Damit geriet der Vorsitz in Konflikt insbesondere zur französischen Regierung, die eine Abkehr von dem Agrarkompromiss aus dem Jahre 2002 kategorisch ablehnte. ${ }^{54}$ Der vierte zentrale Konfliktgegenstand betraf schließlich den so genannten Britenrabatt, einen 1984 von Margaret Thatcher ausgehandelten Ausgleichsmechanismus, nach dem Großbritannien etwa zwei Drittel der Differenz zwischen seinen Aufwendungen für den europäischen Haushalt und seinen Rückflüssen aus diesem Haushalt erstattet bekommt. ${ }^{55}$ Die britische Regierung sah sich bei diesem Verhandlungs-

52 Vgl. Matthew Campbell/Nicola Smith: „Blair's no help, say EU heads“, in: The Sunday Times, 23.10.2005, S. 22.

53 Vgl. UK Presidency of the EU 2005: European Union Financial Perspectives 2007-13 - United Kingdom Presidency Proposal, 5.12.2005, abrufbar unter: http://www.eu2005.gov.uk/servlet/Front?pagename=OpenMarket/Xcelerate/ShowPage \&c=Page\&cid=1112704221230\&a=KArticle \&aid=1133771693498\&date=2005-1205 (letzter Zugriff: 29.12.2005).

54 Vgl. James Blitz/Daniel Dombey/George Parker: „Brown warns French on farms policy“, in: Financial Times, 22.11.2005, S. 2.

55 Zur Einführung des Mechanismus im Juni 1984 vgl. Stephen George: An Awkward Partner. Britain in the European Community, 3. Aufl., Oxford 1998, S. 149-165. 
punkt der geschlossenen Kritik der übrigen 24 Mitgliedstaaten gegenüber, die den Rabatt von jährlich mittlerweile etwa fünf Milliarden Euro als unzeitgemäß ablehnen und dessen substanzielle Kürzung in Vorbereitung seiner mittelfristigen Abschaffung einforderten. Gerade als starker Befürworter der Osterweiterung müsse die britische Regierung nunmehr auch einen angemessenen Teil der Kosten dieser Erweiterung tragen. Diesem Verhandlungsdruck versuchte die britische Präsidentschaft mit ihrem Kompromisspapier vom 5. Dezember Rechnung zu tragen, in dem sie gegenüber der aktuellen Regelung eine Verringerung des Rabatts im Umfang von acht Milliarden Euro über die gesamte Planungsperiode anbot. ${ }^{56}$ Allerdings entsprach dieses Zugeständnis bei Weitem nicht den Juni-Vorschlägen der luxemburgischen Präsidentschaft und wurde von ihren Verhandlungspartnern als unzureichend abgelehnt.

Diese schwierige Konfliktkonstellation ähnelt den Ausgangsbedingungen der deutschen Ratspräsidentschaft 1999, der es im Spannungsfeld zwischen Gesamtumfang der Ausgaben, Finanzierung der Osterweiterung und Reform der Struktur- und Agrarpolitik gelungen war, ein Kompromisspaket über die Finanzielle Vorausschau 2000-2006 zu schnüren. Mit dem Anliegen der Bundesregierung, das Problem der deutschen Nettozahlerposition zu entschärfen, ohne damit die Erweiterungsfähigkeit der Union zu gefährden, gehörte auch in diesen Verhandlungen ein spezifisches Eigeninteresse des Ratsvorsitzes zu den zentralen Parametern des Konflikts. Da dieses Interesse jedoch von den übrigen Nettozahlern in den europäischen Haushalt geteilt wurde, war die deutsche Präsidentschaft im Gegensatz zur britischen Regierung in der Frage ihres Beitragsrabatts auf europäischer Ebene nicht isoliert. ${ }^{57} \mathrm{Im}$ Vergleich dieser beiden Konstellationen, mussten die Aussichten auf die Vermittlung einer Kompromisslösung über die Vorausschau 2007-2013 unter britischem Vorsitz somit schon aus dieser Perspektive zusätzlich geschmälert erscheinen. ${ }^{58}$ Dies galt umso mehr, weil der Handlungsspielraum New Labours hinsichtlich des Britenrabatts durch starke innenpolitische Restriktionen besonders eng beschränkt war. Wie kein anderes Thema dominierte dieser Gesichtspunkt die britische Medienberichterstattung über die Finanzverhandlungen und rückte damit in den Mittelpunkt der öffentlichen Aufmerksamkeit.

An vorderster Stelle bezeichnete die konservative Opposition den Rabatt als unverhandelbares nationales Interesse Großbritanniens. ${ }^{59}$ Unterstützt wurde sie dabei vor allem von den auflagenstärksten Blättern der Boulevardpresse, die bereits die moderaten Zugeständnisse der Regierung Blair in ihrem ersten Verhandlungspapier als „Verrat“ und „Kapitulation“ brandmarkten. ${ }^{60}$ Auch innerhalb des Regierungslagers stand Tony Blair unter erheblichem Druck insbesondere von Finanzminister Gordon Brown, der sich explizit gegen einseitige Kompromisse beim Beitragsrabatt aussprach. ${ }^{61}$

In dieser Ausgangslage strebte die britische Regierung zwar nach einer Ausweitung ihres innenpolitischen Handlungsspielraums, indem sie unpopuläre Konzessionen in der Frage des Britenrabatts zunächst an die populäre Forderung einer substanziellen Verkleinerung des europäischen Agrarhaushaltes knüpfte. Parallel dazu versuchte sie, auf europäischer Ebene Verhandlungsmacht für eine solche Paketlösung aufzubauen, indem sie für den Fall weitge-

56 Vgl. UK Presidency of the EU 2005: European Union Financial Perspectives 2007-13 - United Kingdom Presidency Proposal, 5.12.2005.

57 Vgl. Christoph Jessen: Agenda 2000: Das Reformpaket von Berlin, ein Erfolg für Gesamteuropa, in: integration, 3/99, S. 167-175.

58 Vgl. David Metcalfe: Leadership in European Union Negotiations, 1998, S. 420-422.

59 Vgl. Graham Brady: Blair's desperate EU dash will not save British presidency, Press Release, 1.12.2005, abrufbar unter: http://www.conservatives.com/tile.do?def=news.story.page\&obj_id=126654 (letzter Zugriff: 29.12.2005).

60 Vgl. Benedict Brogan: „The Great Betrayal“, in: Daily Mail, 2.12.2005, S. 2; George Pascoe-Watson: Surrender, in: The Sun, 1.12.2005, S. 2.

61 Vgl. „Stale Summit“, in: The Daily Telegraph, 22.11.2005, S. 19. 
hender Zugeständnisse Großbritanniens und angesichts ihrer fragilen parlamentarischen Machtbasis auf ein mögliches Scheitern der innerstaatlichen Ratifikation einer europäischen Vereinbarung verwies. ${ }^{62}$ Letztendlich stand New Labour auf dem abschließenden Europäischen Rat ihrer Präsidentschaft am 15./16. Dezember 2005 jedoch unverändert vor dem Dilemma, sich entweder auf europäischer Ebene dem Vorwurf mangelnder Kompromissbereitschaft auszusetzen und für ein etwaiges Scheitern der Verhandlungen verantwortlich gemacht zu werden, oder auf innenpolitischer Ebene Kritik für eine zu kompromissbereite Verhandlungsführung auf sich zu ziehen.

Vor diesem Hintergrund ist der auf dem Europäischen Rat von Brüssel vereinbarte Kompromiss über die Finanzielle Vorausschau 2007-2013 umso bemerkenswerter. ${ }^{63}$ Die Verhandlungslösung beruht im Kern auf drei Pfeilern. Erstens wurde das Gesamtvolumen der Vorausschau gegenüber dem ursprünglichen Entwurf der britischen Präsidentschaft um 15 Milliarden Euro auf nunmehr etwa 862 Milliarden Euro ausgeweitet und liegt nur noch neun Milliarden Euro unter dem Vorschlag des luxemburgischen Vorsitzes. Dadurch konnten die Haushaltsmittel für die europäische Strukturpolitik um etwa sieben Milliarden Euro erhöht werden, so dass die finanziellen Einbußen der neuen Mitgliedstaaten Mittel- und Osteuropas im Vergleich zum luxemburgischen Entwurf stark reduziert wurden. Diese Vergrößerung der finanziellen Verteilungsmasse geht zu Lasten der Nettozahler in den europäischen Haushalt und beruht nicht zuletzt auf Zugeständnissen der deutschen Bundesregierung. Zweitens stimmte die französische Regierung einer Klausel zu, die auf der Basis eines Kommissionsberichtes für 2008 oder 2009 die Überprüfung der gesamten europäischen Ausgaben inklusive des Agrarhaushaltes verlangt. Allerdings lässt der Kompromiss das Niveau der Agrarausgaben bis dahin unberührt. Drittens verzichtete die britische Regierung im Vergleich zu ihrem ersten Angebot auf weitere 2,5 Milliarden Euro ihres Beitragsrabatts, der über die gesamte Finanzplanungsperiode somit um 10,5 Milliarden Euro oder etwa 20 Prozent abgeschmolzen wird. Damit bleibt die Gesamtbelastung des britischen Haushalts noch immer um etwa 13 Milliarden Euro geringer als unter den Bedingungen des luxemburgischen Kompromisspapiers, das New Labour im Juni 2005 abgelehnt hatte.

Der erfolgreiche Abschluss der Verhandlungen wird nach den harten Auseinandersetzungen und der zum Teil äußerst massiven Kritik am Vorgehen des britischen Vorsitzes jedoch weniger Tony Blair als der deutschen Bundeskanzlerin angerechnet, die europaweit für ihre Rolle als Vermittlerin und für ihre Kompromissbereitschaft gepriesen wurde. Auf innenpolitischer Ebene entstanden New Labour aus dem Kompromiss über die Finanzielle Vorausschau währenddessen erhebliche politische Kosten. So wurde die Regierung Blair von der europaskeptischen Presse erwartungsgemäß in schärfster Form angegriffen. ${ }^{64}$ Mittelfristig gefährden die Zugeständnisse New Labours in der Frage des Britenrabatts ihre wahlpolitische Unterstützung durch die Murdoch-Presse, die in den letzten drei Unterhauswahlen zu den Erfolgen der Labour-Partei beigetragen hatte. Ebenso erwartungsgemäß hat die konservative Opposition das Verhandlungsergebnis genutzt, um durch pointierte Kritik an der Regierung politisches Kapital zu schlagen. ${ }^{65}$ New Labour hat sich erneut verwundbar gegenü-

62 Vgl. Stephen Castle/Andrew Grice: „Ministers risk parliamentary defeat if EU rebate is reduced, Straw says“, in: The Independent, 22.11.2005, S. 20.

63 Vgl. Council of the European Union: Financial Perspective 2007-2013, 15915/05, Brussels 19 December 2005, abrufbar unter: http://ue.eu.int/ueDocs/cms_Data/docs/pressData/en/misc/87677.pdf (letzter Zugriff: 29.12.2005).

64 Vgl. Michael Lea: „Blair’s the £7bn loser“, in: The Sun, 17.12.2005, S. 2; „Betrayed by Blair“, in: The Daily Telegraph 18.12.2005, S. 22.

65 Vgl. David Cameron: Statement to the House of Commons, in: House of Commons Hansard Debates, 19.12.2005, vol. 440, cols. 1565-1567. 
ber der europaskeptischen Positionierung der konservativen Partei gemacht und damit eine Flanke des politischen Wettbewerbs wiedereröffnet, die sie in den letzten Jahren mühsam geschlossen hielt. Gerade zu einem Zeitpunkt, an dem die konservative Partei unter ihrem neuen Vorsitzenden David Cameron in Umfragen wieder etwa gleichauf mit der LabourPartei liegt, ${ }^{66}$ bietet ihr die Debatte über den Britenrabatt eine zusätzliche Profilierungschance. Die kurzfristig signifikantesten innenpolitischen Kosten des europäischen Kompromisses sind jedoch innerhalb des Regierungslagers auszumachen. Die kaum verdeckte Kritik von Regierungsmitgliedern an der Vereinbarung ist ein weiteres Indiz für den schwindenden Rückhalt Blairs innerhalb seiner eigenen Regierung. Insbesondere Gordon Brown gilt als scharfer interner Kritiker der Verhandlungsführung von Tony Blair, nicht zuletzt weil er sich in den abschließenden Verhandlungen in Brüssel von Blair bewusst übergangen sieht und als Finanzminister Ausgabenpläne der Regierung kürzen muss, um die vereinbarten Mehraufwendungen für die Europäische Union auszugleichen. ${ }^{67}$ Der seit Langem schwelende Machtkonflikt zwischen Blair und seinem potenziellen Nachfolger Brown gewinnt dadurch zusätzliche Brisanz und Sprengkraft. Der innenpolitische Preis, den die Regierung Blair für den Kompromiss über die Finanzielle Vorausschau zahlen muss, ist somit in mehrerer Hinsicht sehr hoch.

\section{Bilanz}

In der Gesamtschau ist es der britischen Präsidentschaft je nach Politikfeld in unterschiedlichem Maße gelungen, sowohl den hohen Erwartungen auf europäischer Ebene als auch den innenpolitischen Anforderungen an ihre Europapolitik gerecht zu werden. So ist für ihre Rolle als Impulsgeber der europäischen Integration eine gemischte Bilanz zu ziehen. Von den drei Themenbereichen, die der britische Vorsitz in den Mittelpunkt seiner Agenda gestellt hatte, konnte er vor allem in der Antiterrorpolitik und in den Außenbeziehungen der Europäischen Union eigene Akzente in Gestalt konkreter Vereinbarungen setzen. Demgegenüber waren die politischen Gegensätze zwischen den Mitgliedstaaten in der Wirtschaftsund Sozialpolitik zu groß, als dass die britische Regierung nennenswerte Erfolge bei der Umsetzung ihrer Reformagenda vorweisen könnte, die über schon im Vorhinein unkontroverse Beschlüsse hinausgehen. Unabhängig von dieser uneinheitlichen Bilanz ist die Politik des britischen Ratsvorsitzes deutlicher Ausdruck der Fähigkeit von Präsidentschaften, die Agenda der europäischen Integration für die Dauer ihrer Amtszeit in einer Weise zu strukturieren, die ihren innenpolitischen Handlungsbedingungen entspricht. Einerseits stellen sich die drei Schwerpunktbereiche der britischen Präsidentschaft im Kern als Transfer der innenpolitischen Agenda New Labours auf die europäische Ebene dar. Mit der partiellen Ausnahme der Dienstleistungsrichtlinie hat die britische Regierung gerade solche Themen aktiv voranzutreiben versucht, die innenpolitisch wenig kontrovers waren. Umgekehrt wirkte die Präsidentschaft einer Debatte im Rat über die Zukunft der Europäischen Verfassung entgegen, die ihr aus innenpolitischer Perspektive nicht opportun erscheinen konnte.

Die Bilanz ihrer Rolle als Konfliktlöser ist in erster Linie durch die beiden Themen bestimmt, die während der britischen Präsidentschaft im Zentrum der mitgliedstaatlichen Auseinandersetzung standen und die unter britischem Vorsitz beide in buchstäblich letzter Sekunde zu einem Verhandlungsabschluss gebracht werden konnten. Dabei ist insbesondere

66 Vgl. ICM Research: The Guardian December Poll, 21.12.2005, abrufbar unter: http://www.icmresearch.co.uk/ reviews/2005/Guardian\%20-\%20December/The\%20Guardian\%20Poll\%20-\%20December\%202005.asp (letzter Zugriff: 29.12.2005).

67 Vgl. David Cracknell/David Smith: „Brown fury at Blair EU climbdown“, in: Sunday Times, 18.12.2005, S. 1. 
der fristgerechte Beginn der Beitrittsverhandlungen mit der Türkei als großer Erfolg der Präsidentschaft festzuhalten, für den sie auf innenpolitischer Ebene breite Zustimmung erfuhr und der zugleich den auf europäischer Ebene fast einhellig formulierten Erwartungen entsprach. Angesichts der schwierigen zwischenstaatlichen Verhandlungskonstellation erscheint die Annahme plausibel, dass es gerade das starke Interesse der britischen Regierung an einem Beitritt der Türkei zur Europäischen Union war, das einen wesentlichen Beitrag zur Konfliktlösung auf europäischer Ebene geleistet hat, der von einer Präsidentschaft mit einer anderen Interessenlage nicht in dieser Form zu erwarten gewesen wäre. Demgegenüber ist es dem britischen Vorsitz in den Verhandlungen über die Finanzielle Vorausschau nicht gelungen, den europäischen Druck zu einer Kompromisslösung mit den innenpolitischen Anforderungen an ihre Verhandlungsführung zu vereinbaren. Zwar konnte auf europäischer Ebene ein Verhandlungsergebnis erzielt werden, für das die Regierung Blair auf innerstaatlicher Ebene jedoch erhebliche politische Kosten zu tragen hat. Sollte die weit verbreitete Vermutung zutreffend sein, dass Tony Blair diese Vereinbarung unter anderem zur Stärkung seines europapolitischen Erbes als Premierminister angestrebt hat, ${ }^{68}$ könnte der Zeitpunkt, an dem dieses Erbe von einem Nachfolger angetreten werden muss, dadurch näher gerückt sein.

68 Vgl. beispielsweise James Blitz: „Budget deal next on the list for UK premier“, in: Financial Times, 28.10.2005, S. 4. 